\title{
Laboratory X-ray powder diffraction as a useful tool for identification of pigments and degradation products in portrait miniatures painted on ivory
}

\author{
E. Kočí ${ }^{1}$, P. Bezdička1 , S. Garrappa ${ }^{1}$, D. Hradil ${ }^{1,2}$, J. Hradilová2 ${ }^{2}$ M. Pech ${ }^{2}$ \\ ${ }^{1}$ Institute of Inorganic Chemistry of the Czech Academy of Sciences, ALMA Laboratory, 25068 Husinec-Řež č.p. 1001, Czech Republic \\ ${ }^{2}$ Academy of Fine Arts in Prague, ALMA Laboratory, U Akademie 4, 170 00Praha 7, Czech Republic \\ eva.koci@iic.cas.cz
}

Fully non-invasive multi-analytical approach combining spectroscopic (FT-IR, Raman) and X-ray-based (MA-XRF, XRPD) techniques was used to study a number of miniature portraits from Czech collections.

The portrait miniatures of the late sixteenth to the nineteenth century represent a highly specific and significant field of European fine art. After 1700, ivory plates were introduced and became the most frequent support of the eighteenth and the nineteenth centuries. Watercolour and gouache were the most common techniques; however, the use of oil has also been recorded.

It is the ivory-painted miniatures that are a special challenge for XRPD - not only because the painting layers are very thin and the ivory signal can interfere the signal from the phases in the painting layer, but also because ivory is a hygroscopic material whose dimensions and curvature respond to changes in environmental conditions (temperature, relative humidity) even during the measurements. We have therefore created a special methodological procedure for measuring miniatures painted on ivory, which we plan to present together with the most interesting results. XRPD helped to identify rare pigments, degradation products and even the binder used thanks to the evidence of metal soaps' formation in paint layers.

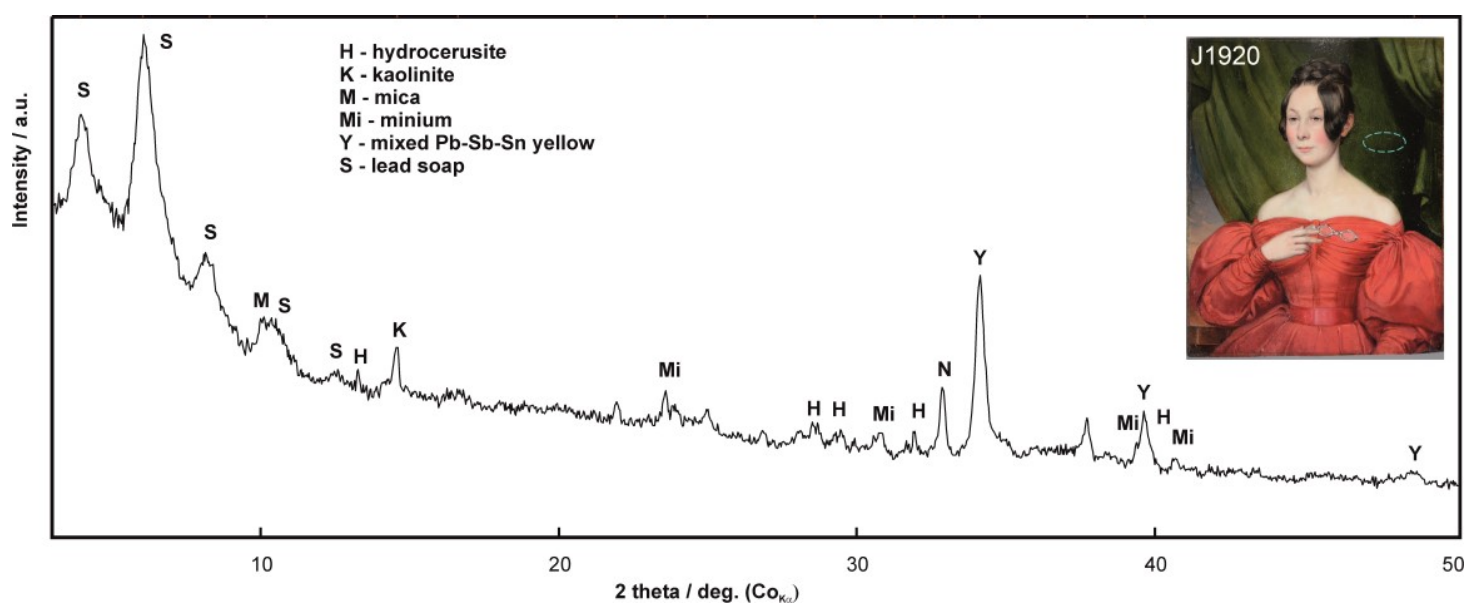

Figure $1 \mathrm{X}$-ray pattern measured in yellow-green curtains in the background. The XRPD identified lead white (H), mixed $\mathrm{Pb}-\mathrm{Sb}-\mathrm{Sn}$ yellow (Y), earth pigments represented by mica (M) and kaolinite (K) and lead soaps (S) formed by interaction of fatty binder (oil) with $\mathrm{Pb}$-based pigments.

\section{Keywords: X-ray diffraction, X-ray microdiffraction, non-invasive analysis, miniature painting, metal soaps}

The study was supported by the Ministry of Culture of the Czech Republic, NAKI II programme, project No. DG18P02OVV034. 